\title{
Association of polymorphism of human $\alpha$ oestrogen receptor gene with coronary artery disease in men: a necropsy study
}

\author{
Tarja A Kunnas, Pekka Laippala, Antti Penttilä, Terho Lehtimäki, Pekka J Karhunen
}

The higher oestrogen concentrations in women have been suggested as the reason for their slower development of atherosclerosis compared with men. Oestrogen receptors have been located on macrophages, smooth muscle cells, and endothelial cells in women and men, but it is not known whether the protective effect at the level of the arterial wall is mediated by these receptors. It has been reported that premenopausal women have fewer $\alpha$ oestrogen receptors in atherosclerotic than in normal coronary arteries. ${ }^{1}$ The gene for human $\alpha$ oestrogen receptor contains a polymorphism in the regulatory (upstream) region of the gene: this polymorphism consists of a dinucleotide (thymine and adenine) repeat, the length of which has been associated with bone mineral density, suggesting an effect on oestrogen receptor transcription. $^{2}$ This prompted us to study whether this polymorphism is associated with coronary artery disease in men.

\section{Subjects, methods, and results}

The associations of the polymorphism with atherosclerosis and myocardial infarction were studied in the Helsinki sudden death study, a prospective series of necropsies of white Finnish men who died suddenly. ${ }^{3}$ Atherosclerotic changes in the coronary arteries were measured by computer assisted planimetry, and coronary narrowings were determined from plastic casts. ${ }^{3}$ The presence of myocardial infarction was confirmed by macroscopic and histological examination of the myocardium. We selected the 119 cases (with mean age 53.4 (SD 8) years) for our analyses according to phenotype -52 men with severe coronary atherosclerosis (mean coronary stenosis $65.9 \%(10.8 \%)$ ) and 67 men with only slightly narrowed coronary arteries (mean 22.1\% (13.7\%)). The causes of deaths were coronary heart disease in 59, violent death or accident in 38 , and other diseases in 22. DNA was extracted from blood by a standard method, amplified by polymerase chain reaction, and analysed by capillary gel electrophoresis as described previously. ${ }^{4}$

Because of the large number of dinucleotide repeats, ${ }^{4}$ we used the median number of the repeat $(n=19)$ to categorise the study population into three groups: those with short allele genotypes (both alleles of $<19$ repeats), those with long allele genotypes (both alleles of $\geqslant 19$ repeats), and those with mixed genotypes (one short and one long allele). In analysis of covariance, with age and body mass index as covariates, men with long allele genotypes had a significantly greater number of severely narrowed coronary arteries $(\mathrm{P}=0.009)$, larger areas of complicated lesions $(\mathrm{P}=0.008)$, and more calcification of the coronary arteries $(\mathrm{P}=0.01)$ than men with short alleles (see figure). Atherosclerotic changes in the mixed genotype group were intermediate and not significantly
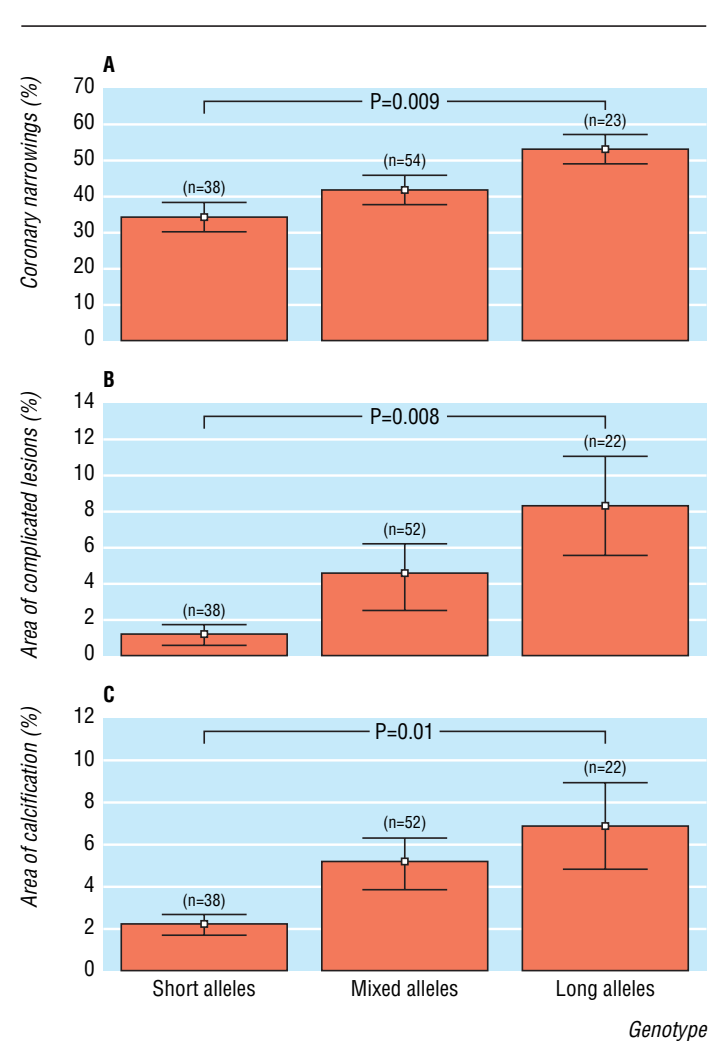

Association of length of oestrogen receptor dinucleotide repeat with coronary narrowing (A), amount of complicated lesions in coronary plaques (B), and area of calcification in coronary arteries (C). Graphs show means (SE), and $P$ values are from analysis of covariance with Scheffe's post hoc test, adjusted for body mass index and age

different from those in the short or long allele genotype group.

A stepwise logistic regression analysis with age and body mass index as covariates showed that the group with long allele genotypes had a higher risk of myocardial infarction compared with the group with short allele genotypes (odds ratio 4.4 (95\% confidence interval 1.21 to 15.70$) ; \mathrm{P}=0.025)$ and seemed to be more predisposed to coronary thrombosis (odds ratio 11.4 (1.2 to 108.8); $\mathrm{P}=0.04$ ). When all confounding factors (age, body mass index, smoking, alcohol consumption, diabetes, hypertension) were forced in statistical models, the tendencies of the results were the same, but the small number of cases with all data $(n=42)$ weakened the statistical significance.

\section{Comment}

Our preliminary results suggest that the length of the dinucleotide repeat in the regulatory region of the $\alpha$ oestrogen receptor gene is associated with severity of
Department of Forensic Medicine, Medical School, FIN-33014 University of Tampere, Tampere University Hospital, Tampere, Finland

Tarja A Kunnas research fellow Pekka J Karhunen professor

School of Public Health, FIN-33014

University of

Tampere

Pekka Laippala professor

Department of Forensic Medicine, PO Box 40, FIN-00014 University of Helsinki, Helsinki, Finland

Antti Penttilä professor

Laboratory of

Atherosclerosis

Genetics,

Department of Clinical Chemistry, FIN-33014

University of

Tampere

Terho Lehtimäki senior research fellow

Correspondence to: T A Kunnas tarja.kunnas@uta.fi

BMJ 2000;321:273-4 
coronary artery disease in men. This may partly explain differences between individuals in the development of coronary artery disease. Of the three other polymorphisms in this gene, the most extensively studied, intronic PvuII polymorphism, showed no association with coronary artery stenosis as measured by angiography. ${ }^{5}$ Although the biochemical evidence is presently lacking, we speculate that carriers of the long repeat variants have lower expression of the oestrogen receptor gene and benefit less from the cardiovascular protective effect of oestrogen receptors.

We thank Outi Lumme and Mervi Niittylahti for their skilful technical assistance, Seppo Tyynelä for planimetric measurements, and Markus Perola for DNA isolation.

Contributors: TAK carried out the genotyping, did most statistical analyses, and wrote the first draft of the article. PL was responsible for statistical analyses. AP was involved in sample and data collection. TL participated in writing the article. PJK initiated the study, was involved in sample and data collection, and is the guarantor for the study. All authors helped in completing the article.
Funding: This work was funded by the Medical Research Fund of Tampere University Hospital, the Yrjö Jahnsson Foundation, the Finnish Foundation of Alcohol Research, and the Tampere Regional Fund of the Finnish Cultural Foundation. Competing interests: None declared.

1 Losordo DW, Kearney M, Kim EA, Jekanowski J, Isner JM. Variable expression of the estrogen receptor in normal and atherosclerotic coronary arteries of premenopausal women. Circulation 1994;8:1501-10.

2 Sano M, Inoue S, Hosoi T, Ouchi Y, Emi M, Shiraki M, et al. Association of estrogen receptor dinucleotide repeat polymorphism with osteoporosis. Biochem Biophys Res Commun 1995;217:378-83.

3 Mikkelsson J, Perola M, Laippala P, Savolainen V, Pajarinen J, Lalu K, et al. Glycoprotein IIIa PIA polymorphism associates with progression of coronary artery disease and with myocardial infarction in an autopsy series of middle-aged men who died suddenly. Arterioscler Thromb Vasc Biol 1999;19:2573-8

4 Kunnas TA, Holmberg-Marttila D, Karhunen PJ. Analysis of estrogen receptor dinucleotide polymorphism by capillary gel electrophoresis with a population genetic study in 180 Finns. Hum Hered 1999;49:142-5.

5 Matsubara Y, Murata M, Kawano K, Koichi Z, Zama T, Aoki N. Genotype distribution of estrogen receptor polymorphism in men and postmenopausal women from healthy and coronary populations and its relation to serum lipid levels. Arterioscler Thromb Vasc Biol 1997;17:3006-12.

(Accepted 14 April 2000)

\title{
Early neonatal mortality, asphyxia related deaths, and timing of low risk births in Hesse, Germany, 1990-8: observational study
}

\author{
Günther Heller, Björn Misselwitz, Stephan Schmidt
}

Institute of Medical Sociology and Social Medicine, Medical Centre of Methodology and Health Research, Philipps-University of Marburg, Medical School, D 35033 Marburg, Germany Günther Heller assistant professor

Department of Obstetrics, Centre of Gynaecology and Obstetrics,

Philipps-University of Marburg

Stephan Schmidt professor of gynaecology and obstetrics

Institute of Quality Assurance Hesse, D 65760 Eschborn, Germany

Björn Misselwitz public health researcher

Correspondence to: G Heller hellerg@mailer. uni-marburg.de

BMJ 2000;321:274-5
A higher neonatal mortality related to intrapartum events during the night has been reported in Great Britain. ${ }^{12}$ We investigated whether the time of birth affects early neonatal mortality or deaths related to asphyxia in low risk births.

\section{Participants, methods, and results}

Data from the perinatal birth register of the federal state of Hesse, Germany, 1990-8, were used (www.medqs-hessen.de). The register comprises detailed information about all infants born in birth clinics (more than $95 \%$ of all births in Hesse); about the mother, including the pregnancy; and about the delivery, as documented by the obstetrician in charge of the birth, using an evaluated standardised questionnaire comprising 67 items. ${ }^{3}$ Detailed information is available about the child's morbidity and reasons for death coded in 40 predefined categories adapted from ICD-9 (international classification of diseases, 9 th revision).

Outcome events were deaths during labour or within the first seven days of life (early neonatal deaths) and asphyxia related deaths during the same period.

Early neonatal mortality and timing of birth in low risk births in Hesse, Germany, 1990-8

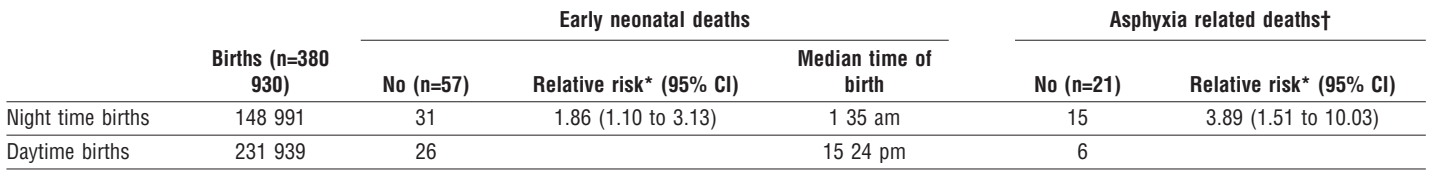

*Births at night $v$ births during the day.

tAsphyxia given as cause of death for infants who died during labour or in the first seven days of life.
Completeness of the record of all early neonatal deaths was validated by comparison with corresponding death rates as reported by the statistical office of Hesse. $^{4}$

To control for the effects of planned births with respect to the time of birth we excluded antepartum deaths, caesarean sections, infants born before the 37 th week of gestation, and infants with congenital malformations or hereditary metabolic diseases. Those births occurring between $9 \mathrm{pm}$ and 659 am were defined as night time births. Otherwise births were assumed to have taken place during the day.

Relative risks and 95\% confidence intervals were calculated to assess the effect of night and day on death rates. A total of 380930 births met the criteria for inclusion. Fifty seven early neonatal deaths were observed, reflecting the low mortality risk of these selected births. Babies born at night were almost twice as likely to die as babies born during the day (relative risk $=1.86 ; 95 \%$ confidence interval 1.10 to 3.13 ). For deaths related to asphyxia an even more pronounced relation was observed $(3.89 ; 1.51$ to 10.03 ; table). 